\section{Evaluation of Prehospital Intravenous Fluid Warming Techniques}

\section{Wipfler EJ, Wolford RW}

Central Illinois Center for Emergency Medicine, Saint Francis Medical Center

Affiliate of University of Illinois College of Medicine Peoria, Illinois, USA

Purpose: Cold-weather prehospital care often is asociated with patient hypothermia which may be exacerbated by infusion of cold intravenous fluid (IVF). Three methods of field IVF warming were studied.

Methods: Techniques of field warming of one-liter D5NS bags tested were: 1) IVF pressure bag (PB) with disposable hot pack; 2) Arctic Intra-Therm (AIT) (Safe \& Warm INC), a soft-fabric insulator with reusable instant heat source; 3) Reusable heat source packs (Zap Pac, PRISM Tech) placed with IVF inside an insulated box (IB) (Coleman 16 Cooler). IVF temperatures were measured using a Fluke Model 52 digital thermometer (accuracy $\pm 0.1^{\circ} \mathrm{C}$ ). The initial IVF temperature was $18^{\circ} \mathrm{C}$. All techniques were evaluated in $\mathrm{a}-15^{\circ} \mathrm{C}$ walk-in freezer and measured in triplicate. Statistical significance was determined using the Repeated Measures Manova model $(p<0.001)$.

Results: The IVF temperature and time to freezing varied significantly and are in the Table:

$\begin{array}{ccccc}\begin{array}{l}\text { IVF Mean } \\ \text { Time (Hrs) }\end{array} & \text { Control } & \text { PB } & \text { AIT } & \text { IB } \\ 0 & 18.0 & 18.0 & 18.0 & 18.0 \\ 0.5 & 9.3 & 16.5 & 24.0 & 32.6 \\ 1.1 & 0 & 11.1 & 25.0 & 35.0 \\ 2.2 & - & 2.4 & 18.7 & 35.5 \\ 2.7 & - & 0 & 17.5 & 37.6 \\ 4.0 & - & - & 12.5 & 37.0 \\ 5.0 & - & - & 6.3 & 0 \\ 7.5 & - & - & 0 & 31.0 \\ 14.0 & - & - & - & 18.8 \\ 25.0 & - & - & - & 5.4 \\ 33.0 & - & - & - & 0\end{array}$

Conclusion: All methods significantly slowed the rate of cooling. The Arctic Intra-Therm and Insulated Box significantly increased IVF temperature in below-freezing conditions. The Insulated Box may provide IVF at near body temperature in a frigid environment.

\section{Discomfort in Healthy Volunteers Immobilized on Wooden Backboards and Vacuum Mattress Splints}

\author{
Delbridge TR, Auble TE, Garrison HG, Menegazzi IJ \\ Division of Emergency Medicine, University of Pittsburgh \\ Pittsburgh, Pennsylvania, USA
}

Purpose: Discomfort experienced by patients immobilized on a wooden backboard is problematic, not only for the suffering patient, but also for the emergency staff who must frequently determine whether experienced pain is due to immobilization or injury. Unnecessary radiographs may be obtained in the process. Recently, a vacuum mattress splint has been marketed as causing less discomfort than standard spinal immobilization techniques. The purpose of this study was to determine if there is a difference in the degree of discomfort experienced by individuals immobilized on a wooden backboard versus a vacuum mattress splint.

Methods: Twelve healthy adult volunteers participated in a randomized crossover study. Participants were immobilized on a backboard and a vacuum mattress splint (Hartwell Medical), each for two hours. Levels of discomfort were recorded by the subjects at regular intervals on $10 \mathrm{~cm}$ visual analog scales, corresponding to anatomic sites that included head, neck, lower back, and overall. The Wilcoxon Sign Rank test with alpha set at $<0.05$ was used to compare scores between devices at each time interval.

Results: The following table provides the median visual analog scale scores $(\mathrm{mm})$ for anatomic regions at the indicated time intervals.

\begin{tabular}{|c|c|c|c|c|c|}
\hline & & $30^{\prime}$ & $60^{\prime}$ & $90^{\prime}$ & $120^{\prime}$ \\
\hline \multirow[t]{2}{*}{ Head } & Backboard & 5.5 & 18.0 & 21.0 & 25.5 \\
\hline & Mattress & 5.2 & $5.8^{*}$ & $5.0^{*}$ & $5.5^{*}$ \\
\hline \multirow[t]{2}{*}{ Neck } & Backboard & 2.5 & 6.5 & 9.0 & 11.0 \\
\hline & Mattress & 2.5 & 7.0 & $6.0^{*}$ & 8.0 \\
\hline \multirow[t]{2}{*}{ Lower Back } & Backboard & 7.5 & 20.5 & 29.5 & 35.0 \\
\hline & Mattress & 1.0 & $6.8^{*}$ & 7.0 & $7.5^{\star}$ \\
\hline \multirow[t]{2}{*}{ Overall } & Backboard & 3.5 & 17.5 & 19.0 & 27.5 \\
\hline & Mattress & 4.0 & 10.5 & $8.0^{*}$ & 12.5 \\
\hline
\end{tabular}

* denotes $p<.05$ for comparison between backboard and mattress ' denotes minutes

Conclusion: The results suggest that vacuum mattress splints cause less discomfort than do wooden backboards; the effect is more pronounced as the duration of spinal immobilization increases. Prospective studies are necessary to evaluate the clinical significance of this effect and the cost-effectiveness of similar alternative immobilization devices. 